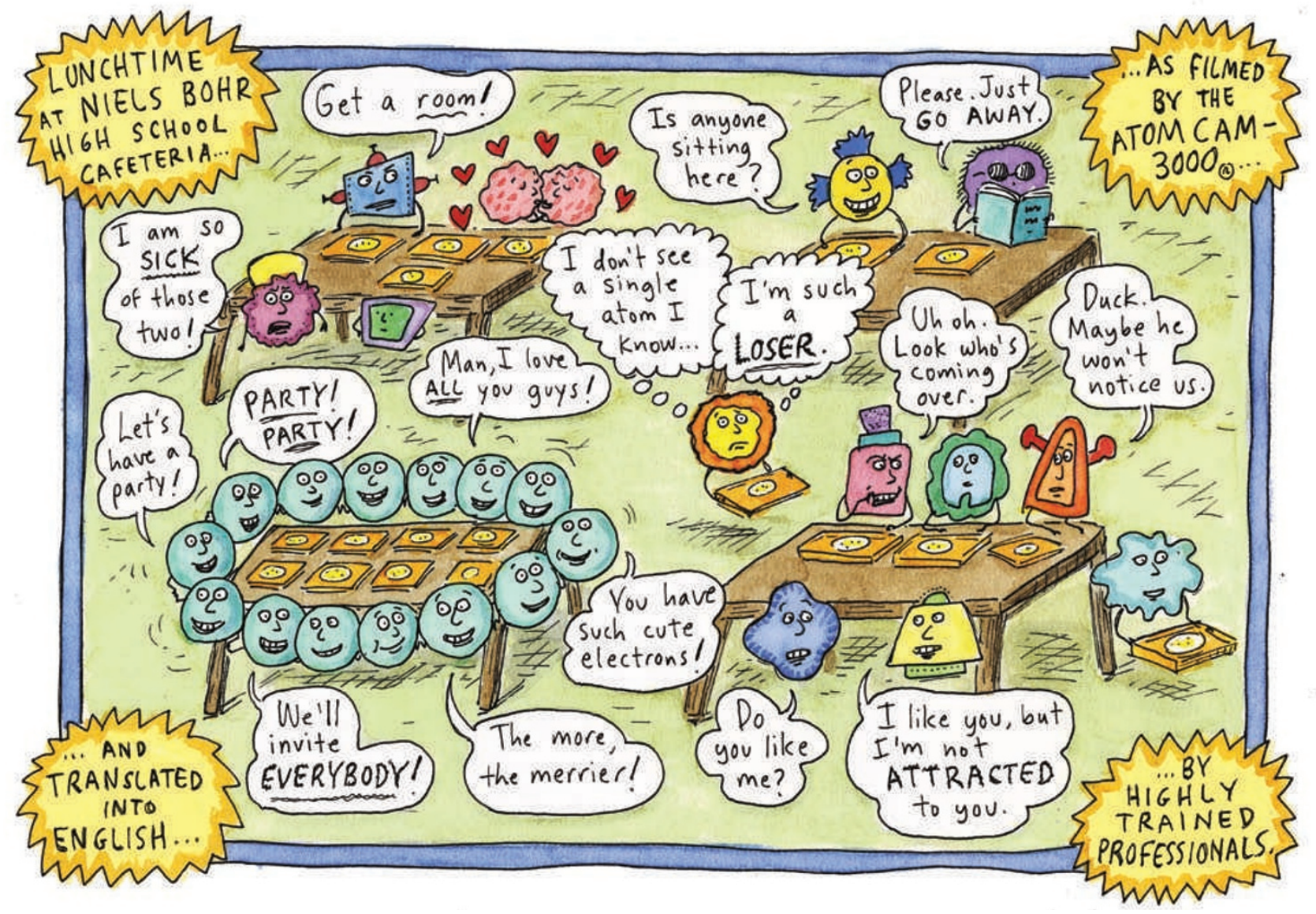

\title{
Beyond the bond
}

\section{More than ever before, new techniques show the bond to be a convenient fiction, albeit one that holds the field of chemistry together, finds Philip Ball.}

$\mathrm{N}$ ot so long ago, the chemistry student's standard text on the theory of chemical bonding was Charles Coulson's Valence (1952). Absent from it was this theoretical chemist's real view on the sticks that generations of students have drawn to link atoms into molecules. "A chemical bond is not a real thing: it does not exist: no one has ever seen it, no one ever can. It is a figment of our own imagination," he later wrote ${ }^{1}$.

There is a good reason for postponing this awkward truth. The bond is literally the glue that makes the entire discipline cohere; to consider it an objective reality is necessary for any kind of discourse on chemistry. The discipline is in fact riddled with such convenient (and contested) fictions, such as electronegativity, oxidation state, tautomerism and acidity.

Disputes about the correct description of bonding have ruffled chemists' feathers since the concept of molecular structure first emerged in the mid-nineteenth century. Now they are proliferating, as new theoretical and experimental techniques present fresh ways to probe and quantify chemical bonds ${ }^{2}$. Traditional measures such as crystallographic interatomic distances and dissociation energies have been supplemented by spectroscopic techniques for determining vibrational frequencies, methods such as nuclear magnetic resonance to measure shifts in the electronic environment of atoms and their magnetic interactions, measurements of force constants (bond stiffness) and a host of quantum-chemical tools for calculating such aspects as electron distributions or localization.

An indication of the shifting ground is the recent decision to redefine the nature of the hydrogen bond in the light of experimental results that alter the traditional electrostatic description.

The nature of the chemical bond is now further complicated by the introduction of

\begin{tabular}{|l|l|}
\hline$a$ & 2011: YEAR OF CHEMISTRY \\
3 & $\begin{array}{l}\text { Celebrating the central science } \\
\text { nature.com/chemistry2011 }\end{array}$ \\
\hline
\end{tabular}

the dynamical dimension. Molecules have traditionally been regarded, if not as static then as having clear architectural frame works that are merely shaken and rotated by thermal motions. The bonds get stretched and bent, but they still have an equilibrium length and strength that seems to justify their being pictured as lines and stalks. Now, thanks to ultrafast spectroscopies, such timeaverage values may not always accurately characterize either structure or reactivity. What is measured in a bond depends not just on how but on when it is measured.

Some chemists argue that as a result of this, the very existence (or not) of a bond depends on how the problem is probed. Others are committed to absolute criteria. This difference of opinion goes to the heart of what chemistry is about: can all be reduced to quantum physics, or are fuzzy rules of thumb essential? More pressingly, the issue of how best to describe a chemical bonding pattern has tangible implications for a wide range of problems in chemistry, from molecules in which atoms are coerced out of their 
usual bonding geometry to the symmetric hydrogen bond (in which a bound hydrogen atom is shared equally between two other atoms), and new variations on old themes such as aromaticity (special patterns of 'delocalized' bonds, like those in benzene) ${ }^{3}$.

Just about every area of chemistry harbours its own bonding conundrums. Most illustrate that we have a far from exhaustive understanding of the ways in which quantum rules will permit atoms to unite - and that in consequence, the synthetic inventiveness of chemists suffers from a limited view of the possibilities.

\section{CARVING UP ELECTRONS}

We can all agree on one thing: chemical bonding has something to do with electrons. Two atoms stick together because of the arrangement of electrons around their nuclei. In the nineteenth century, it was commonly thought that this attraction was electrostatic: that atoms in molecules are positively or negatively ionized. That left the puzzle of how identical atoms can form diatomic molecules such as $\mathrm{H}_{2}$ and $\mathrm{O}_{2}$. American chemist G. N. Lewis proposed that bonding can instead result from the sharing of electrons to create filled shells of eight, visualized as the corners of a cube.

In the 1920s and 1930s another American chemist, Linus Pauling, showed how this interaction could be formulated in the language of quantum mechanics as the overlap of electron orbitals (see Nature 468, 1036; 2010). In essence, if two atomic orbitals each containing a single electron can overlap, a bond is formed. Pauling generalized earlier work on the quantum description of hydrogen to write an approximate equation for the wavefunction created by orbital overlap. This became known as the valence-bond (VB) description.

But an approximation is all it is. Around the same time, Robert Mulliken and Friedrich Hund proposed another approximate wavefunction, which led to an alternative way to formulate bonds: not as overlaps between specific orbitals on separate atoms, but as electron orbitals that extend over many atoms, called molecular orbitals (MOs). The relative merits of the VB and MO descriptions were debated furiously for several decades, with no love lost between the protagonists: Mulliken's much-repeated maxim, "I believe the chemical bond is not so simple as some people seem to think", was possibly a jibe at Pauling. By the 1960s, for all Pauling's salesmanship, MO theory was generally agreed to be more convenient for most purposes. But the debate is not over ${ }^{4}$, and Roald Hoffmann of Cornell University in Ithaca, New York, insists that "discarding any one of the two theories undermines the intellectual heritage of chemistry".

Both options are imperfect, because they insist on writing the electronic wavefunction as some combination of the wavefunctions of individual electrons. That's also the basis of the Hartree-Fock method for calculating the wavefunction and energy of the lowestenergy state (ground state) of a molecular system - a method that became practical in the 1950s, when computers made it possible to solve the equations numerically. But separating the wavefunction into oneelectron components is a fiction, as they all influence one another: the behaviour of one electron depends on what all the others are doing. The difference between true ground-state energy and that calculated using the Hartree-Fock approach is called the correlation energy. More recent computational methods can capture most of the correlation energy, but none can give an exact solution. As a result, describing the quantum chemical bond remains a matter of taste: all descriptions are, in effect, approximate ways of carving up the electron distribution.

If that were the limit of the bond's ambiguity, there would be little to argue about. It is not. There is, for example, the matter of when to regard two atoms as being bonded at all. Pauling's somewhat tautological definition rather gave the game away: a group of atoms is to be considered bonded when it is "convenient for the chemist to consider it as an independent molecular species". Pauling admitted some of the consequent ambiguities: for example, although his

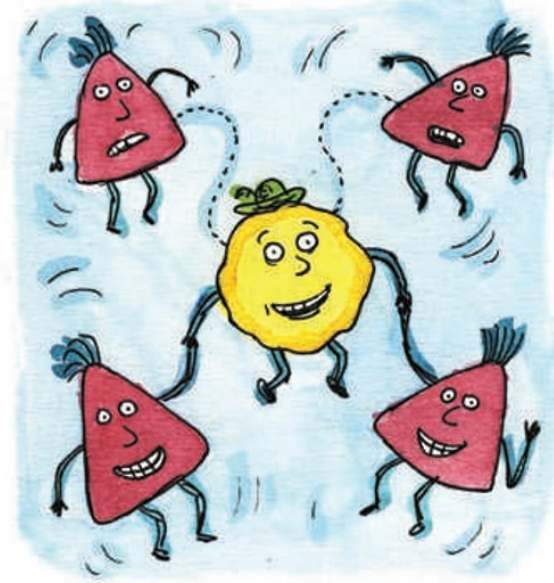

definition in general excludes the weak van der Waals attraction that occurs between all atoms, occasionally - as in the association of two oxygen molecules into the $\mathrm{O}_{4}$ cluster - even this force can be strong enough to be regarded as a chemical bond.

It's no use, either, to suggest (as Coulson did) that a bond exists whenever the combined energy of the atoms is lower than that when they are separated by an infinite distance. For this is essentially always the case, at least for electrically neutral atoms. Even two helium atoms experience mutual van

der Waals attraction, which is why helium is a liquid at very low temperatures; but they are not generally thought to be chemically bonded as a result.

Besides, the 'bonded or not' question becomes context-dependent once atoms are embedded in a molecule, where they may be forced close together merely by the atoms around them, and where there is inevitably some arbitrariness in decid-

ing which electrons

"Chemists' inventiveness suffers from alimited view of the possibilities."

'belong' to which atoms. The resulting ambiguities were illustrated recently when three experts failed to agree about whether two sulphur atoms in an organometallic compound are linked by a bond ${ }^{5}$. The argument involved different interpretations of quantum-chemistry calculations, tussles over the best criteria for identifying a bond, and evidence of precedent from comparable compounds.

All this is merely a reminder that a molecule is ultimately a set of nuclei embedded in a continuous electron cloud that stabilizes a particular configuration, which balls and sticks can sometimes idealize and sometimes not. But disputes about the nature of the chemical bond are not simply semantic. It matters, for example, whether we regard a very strong multiple bond as quintuple or sextuple, even if this is a categorization that only textbooks, and not nature, recognize - not least, because textbook concepts are what provide a discipline with intellectual coherence and consistency.

Besides, how we talk about bonds can determine our ability to rationalize real chemical behaviour. For example, the different descriptions of the bonds in what are now called non-classical ions of hydrocarbons whose relative merits were furiously debated in the 1950s and 1960s - have direct implications for the way these species are predicted to react. Whether to consider the bonding non-classical, involving electrons spread over more than two atomic nuclei, or tautomeric, involving rapid fluctuations between conventional two-atom bonds, had immediate consequences for organic chemistry.

Perhaps one might seek a distinction between bonded and not-bonded in terms of how the force between two atoms varies with their separation? Yes, there is an exponential fall-off for a covalent bond such as that in $\mathrm{H}_{2}$, and a power-law decay for van der Waals attraction. But the lack of any clear distinction between these two extremes has been emphasized in the past two decades by the phenomenon of aurophilicity ${ }^{6}$, in which gold atoms that have only a few chemical groups attached to them in organometallic compounds tend to aggregate, forming dimers 


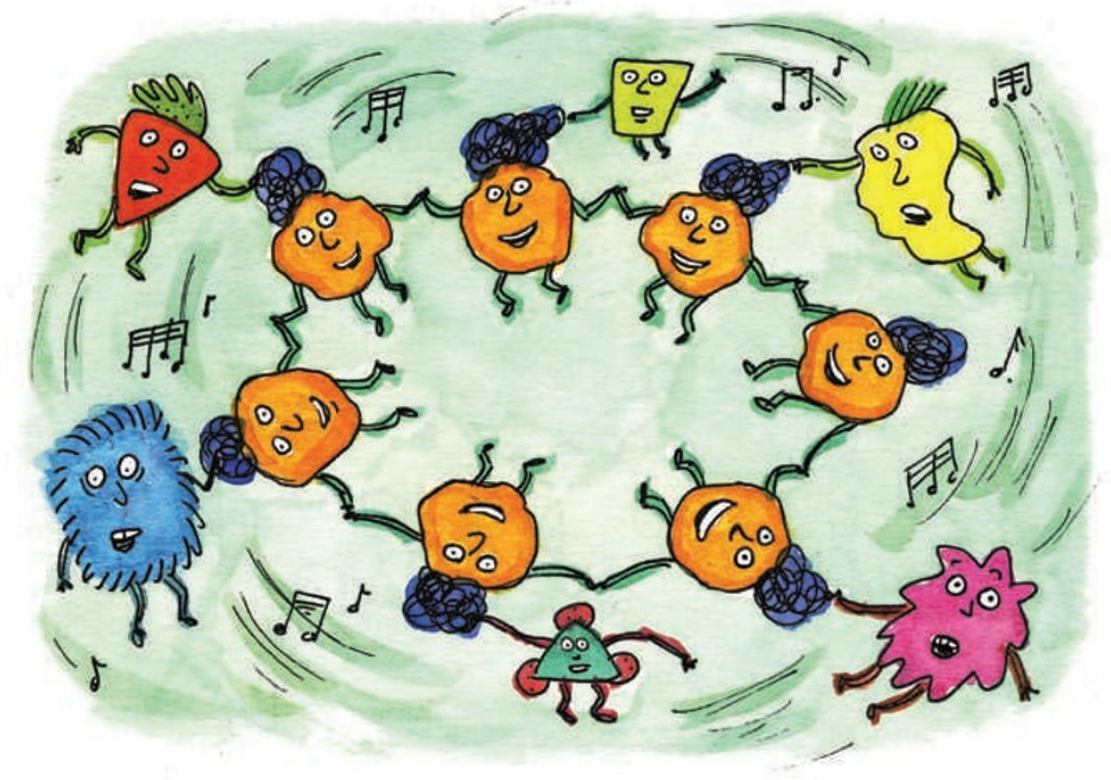

and linear chains. The basic interaction in these aurophilic bonds between gold has the same origin as the van der Waals force: the electron clouds 'feel' each other's movements, so that random fluctuations of one induce mirror-image fluctuations of the other. But that interaction is modified here by relativistic effects: changes in electron energies resulting from their high speeds around gold's highly charged, massive nuclei ${ }^{6,7}$. Aurophilic bonds have therefore been described as a 'super van der Waals' interaction. Does that make them true bonds? It's chemically meaningful to treat them that way (they'll even serve for cementing new 'designer' molecular crystals ${ }^{8}$ ), but perhaps at the cost of relinquishing another potential criterion for 'real' bonds.

\section{MOVING TARGET}

Perhaps the biggest challenge to the simple notion of a chemical bond is dynamics. Atomic motions make even a 'simple' molecule complex; any movement of one nucleus demands that the entire electron cloud adjusts. A jiggle of one group of nuclei can make it easier to cleave off another.

This complication never used to matter much in chemistry, because the movements were too rapid to be observable, much less exploitable. Ultrashort pulsed lasers moved the goal posts. For example, energy can be pumped into a vibrational mode to weaken a specific bond, enabling selective molecular surgery ${ }^{9}$. Chemists can ask about the chemical behaviour of a molecule at a particular moment in its dynamical evolution. Even a strong bond is weakened when a vibration stretches it beyond its average, equilibrium length, so in ultrafast chemistry it may no longer be meaningful to characterize bonds simply as strong or weak. As Fleming Crim of the University of Wisconsin-Madison puts it: "A bond is an entity described by quantum mechanics but not a fixed 'entity' in that it will behave differently depending on how we perturb and interrogate it." The trajectory of a chemical reaction must then be considered not as a making and breaking of bonds but as an evolution of atoms on a potential-energy surface. This was always implicit in classical drawings of transition states as molecular groupings containing dashed lines, a kind of 'almost bond' in the process of breaking or forming. Now that picture is explicitly revealed as a mere caricature of a complicated dynamical process in space and time.

Underlying most of these discussions is an unspoken assumption that it is meaningful to speak, if not of a 'bond' as an unchanging entity, then at least of an instantaneous bound

$\begin{array}{ll}\text { "The biggest } & \text { state for a particular } \\ \text { challenge } & \text { This assumes that the } \\ \text { to the simple } & \text { electrons can adjust } \\ \text { notion of } & \text { more or less instantly } \\ \text { abond is } & \text { to any change in the } \\ \text { dynamics." } & \text { nuclear positions: the } \\ & \text { Born-Oppenheimer }\end{array}$

approximation. Because electrons are so much lighter than nucleons, this assumption is usually justified. But some clear breakdowns of the approximation are now well documented $^{10}$. They are best known in solidstate systems, and in fact superconductivity is one of the consequences, resulting from a coupling of electron and nuclear motions. Such things may also happen in molecules, particularly in the photochemistry of polyatomic molecules, which have a large number of electronic states close in energy ${ }^{11}$; they have also been observed for simple diatomic molecules in strong electric fields ${ }^{12}$. As a result, the molecular degrees of freedom may become interdependent in strange ways: rotation of the molecule, for example, can excite vibration. In such situations, the very notion of an electronic state begins to crumble ${ }^{10}$.
These advances in dynamical control of quantum states amount to nothing less than a new vision of chemistry. The static picture of molecules with specific shapes and bond strengths is replaced by one of a bag of atoms in motion, which can be moulded and coaxed into behaviours quite different from those of the equilibrium species. Such a vision does not demand that we abandon old ideas about chemical bonds, nor does it truly challenge the ability of quantum theory to describe atoms and their unions. It recommends that we view these bonds as degrees of attraction that wax and wane - or as cartoon representations of a molecule's perpetual tour of its free-energy landscape.

At a meeting in 1970, Coulson asserted that the simple notion of a chemical bond had already become lost, and that "something bigger" was needed to replace it - a subject, he suggested, for another meeting 50 years hence. That moment is almost upon us.

Yet we needn't fret that the 'rules' of bonding are up for grabs - quite the reverse. Although there may be some parts of science fortunate enough to be exhaustively explained by a single, comprehensive theory, this isn't likely to be a general attribute. We are typically faced with several theories, some overlapping, some conflicting, some just different expressions of the same thing. Our choice of theoretical framework might be determined less by the traditional criterion of consistency with experiment than by subjective reasons. According to Hoffmann, these preferences often have an aesthetic component, depending on factors such as simplicity, utility for telling a story about chemical behaviour, the social needs of the community and whether a description is productive.

As Hoffmann says: "Any rigorous definition of a chemical bond is bound to be impoverishing." His advice to "have fun with the fuzzy richness of the idea" is well worth heeding.

Philip Ball is a writer based in London.

1. Coulson, C. A. The Spirit of Applied Mathematics 20-21 (Clarendon Press, 1953).

2. J. Comput. Chem. special issue $\mathbf{2 8 , 1 - 4 6 6}$ (2007).

3. Cortés-Guzmán, F. \& Bader, R. F. W. Coord. Chem. Rev. 249, 633 (2005).

4. Hoffmann, R., Shaik, S. \& Hiberty, P. C. Acc. Chem Res. 36, 750-756 (2003).

5. Alvarez, S., Hoffmann, R. \& Mealli, C. Chem. Eur. J. 15, 8358-8373 (2009).

6. Pyykkö, P. Chem. Soc. Rev. 37, 1967-1997 (2008).

7. Schmidbaur, H., Cronje, S., Djordjevic, B. \& Schuster, O. Chem. Phys. 311, 151-161 (2005).

8. Katz, M. J., Sakai, K. \& Leznoff, D. B. Chem. Soc. Rev. 37, 1884-1895 (2008).

9. Crim, F. F. Science 249, 1387 (1990)

10.Sukumar, N. Found. Chem. 11, 7-20 (2009).

11. Worth, G. A. \& Cederbaum, L. S. Ann. Rev. Phys. Chem. 55, 127-158 (2004).

12.Sindelka, M., Moiseyev, N. \& Cederbaum, L. S. preprint at www.arxiv.org/abs/1008.0741 (2010). 